\title{
Adhesive contribution to friction
}

Cite as: AIP Conference Proceedings 2167, 020286 (2019); https://doi.org/10.1063/1.5132153

Published Online: 19 November 2019

V. L. Popov

\section{ARTICLES YOU MAY BE INTERESTED IN}

Regimes of adhesive wear in dry contact: Conditions of realization and determining parameters

AIP Conference Proceedings 2167, 020075 (2019); https://doi.org/10.1063/1.5131942

Mechanics of adhesive contacts: Experiment and theory

AIP Conference Proceedings 2167, 020201 (2019); https://doi.org/10.1063/1.5132068

Active bio contact mechanics: Concepts of active control of wear and growth of the cartilage in natural joints

AIP Conference Proceedings 2167, 020285 (2019); https://doi.org/10.1063/1.5132152

\section{Lock-in Amplifiers up to $600 \mathrm{MHz}$

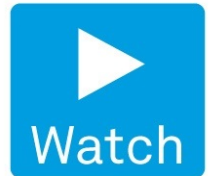

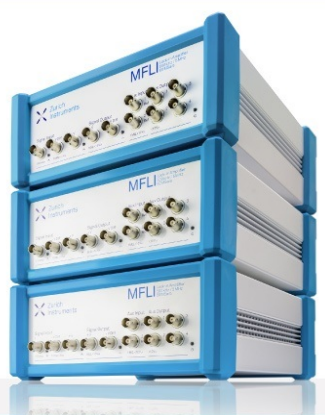




\title{
Adhesive Contribution to Friction
}

\author{
V.L. Popov ${ }^{\mathrm{a})}$ \\ Technische Universität Berlin, Berlin, 10623 Germany \\ ${ }^{\text {a)} C o r r e s p o n d i n g ~ a u t h o r: ~ v . p o p o v @ t u-b e r l i n . d e ~}$
}

\begin{abstract}
This paper addresses the question: what is the physical origin of friction in a contact of two amorphos, hard, smooth elastic surfaces (as e.g. DLC coatings). The dissipation channels due to viscoelasticity, plasticity or elastic instabilities are not active in such systems. We show that short range adhesion leads to "adhesion instabilities" which cause energy dissipation similarly to the classic elastic instabilities mechanicsm. We argue that the main governing parameter determining friction in such systems is the "Johnson parameter" introduced 1995 by K.H. Johnson.
\end{abstract}

\section{INTRODUCTION}

Friction is a very important phenomenon, which enigineers would like to be able to control, to increase or decrease at our will [1]. But it is also a phenomenon which is still poorly understood. Friction means energy dissipation. Searching for mechanisms of friction we try to answer the question: where the mechanical energy does disappear? In a lubricated contact, it is the viscosity which leads to energy losses. But the fluid friction cannot support static loads. Lubricated contacts are well understood but not the subject of this paper. In elastomer materials as rubber, it is the viscoelaticity, which leads to energy losses. In this case, the contact is asymmetric, due to viscosity, and this is the physical reason for appearance of the force of friction. Very similar mechanism is due to plasticity instead of viscoelasticity. This mechanism was very schematically illustrated described in the famous book by Bowden and Tabor [2]. Here too, the force of friction appears due to asymmetry of the contact due to plastic deformation. But what is the physical origin of friction in a contact of hard elastic solids?

This question was treated 1928 in a paper by the famous Ludwig Prandtl who developed a very simple model explaining the mechanism of energy dissipation in the case when the system is purely elastic $[3,4,5]$. As a matter of fact, Prandtl used it as a model of plasticity and only mentioned briefly that it is even better applicable for describing friction. Many years later, the Prandtl model became a standard model in interpreting experiments on nanoscale friction between a tip of an atomic force microscope and atomically smooth surfaces such as graphite or mica [6].

In the 1990s there was a true rediscovery of tribology. In this time, many fundamental aspects of friction on the nanoscale were correctly understood and proved experimentally. Among the most fundamental understandings was the essential importance of elastic instabilities as described by Prandtl. If a tip of an atomic force microscope is forced to slowly move along an atomically flat surface, than the macroscopic force of friction, that is tangential force averaged over some macroscopic distance, is exactly zero, provided the cantilever of the AFM is stiff enough. In other words, if the tip follows continuously the moving stage, there is no friction in the system. The situation changes qualitatively if the stiffness is smaller than some critical value. Then the tip does not follow the stage continuously any more but shows sudden jumps. And these are only these jumps, known as elastic instabilities, which cause irreversible energy dissipation - in form of waves going away from the contact region [7]. In Fig. 1 results of experimental measurements of lateral force of an atomic force microscope moving forth and back in the overcritical state is shown, with jumps and the under critical state, without jumps as well as simulation of the corresponding movement with the Prandtl model [8]. Energy dissipation in one complete cycle is equal to the area between the force displacement curve for forth and back movement. If elastic instabilities occur, both curves do not coincide and there is some energy dissipation. Without jumps, there is no hysteresis and thus no energy losses. One can summarize this by the statement: No elastic instabilities - no friction! 

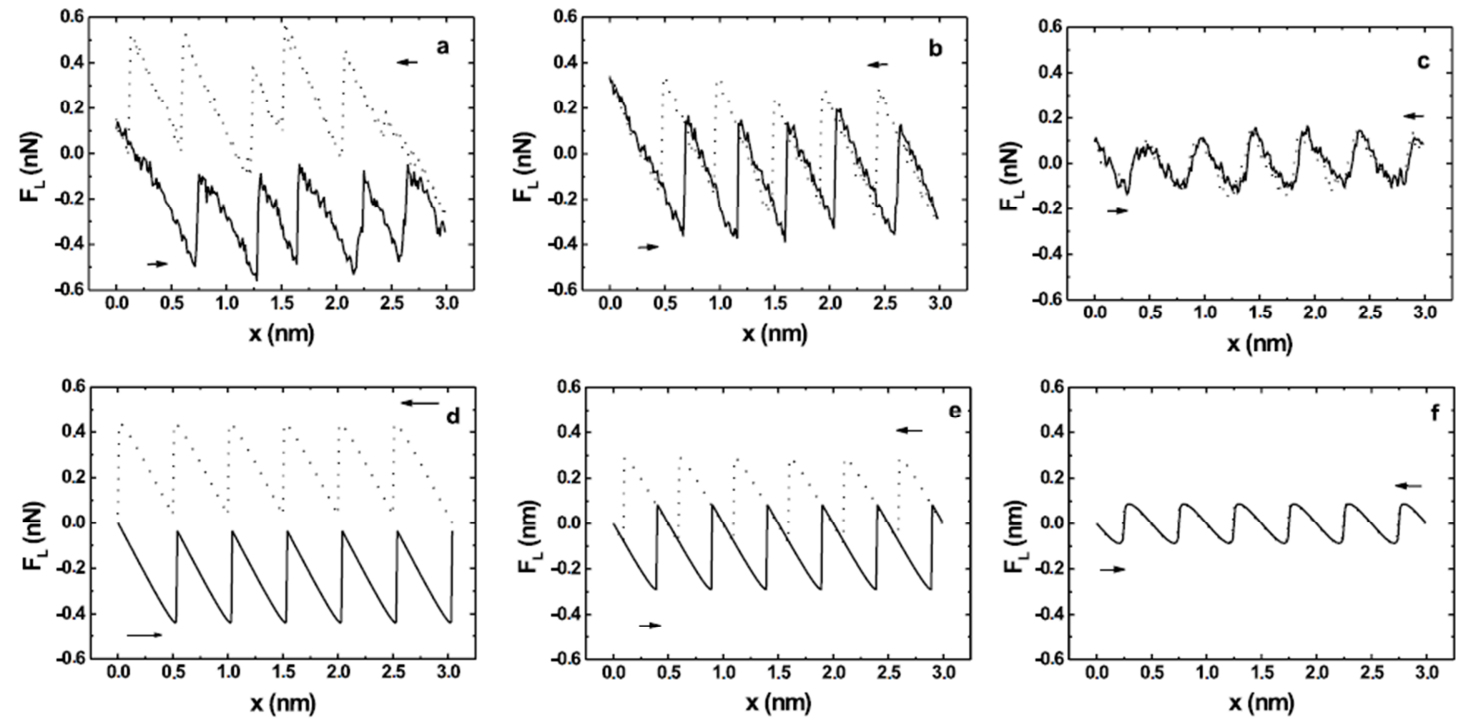

FIGURE 1. Experimental data from [8] (a) -(c) Measurements of the lateral force acting on the tip sliding forward and backward in (100) direction over the $\mathrm{NaCl}(001)$ surface with decreasing normal force. (d) -(f) Corresponding numerical results from the Prandtl-Tomlinson model with suitable parameters. For small values of the normal force, the hysteresis loop enclosed between the forward and the backward scan vanishes; i.e., there is no more dissipation within this model.

The concept of elastic instabilities lead further to the concept of structural superlubricity. If two bodies with atomically smooth periodic surfaces are in contact then final friction appears only if they have equal or similar periods. In the case of incommensurate lattice periods, the friction is vanishing. This is especially easy to illustrate by rotation one crystalline body relatively to the other one. In commensurate orientation, there is high friction. And friction practically disappears if the are not oriented so that they match each other (see Fig. 2). But exactly this fundamental understanding makes so difficult the question of what is the mechanicsm of friction in hard, amorphous, elastic smooth surfaces as we have e.g. in the contact of an DLC coating of the supporting columns with the silicon oxide layer of a silicon wafer what it is lying on the transferring table in a chip manufacturing machine or many other similar systems.

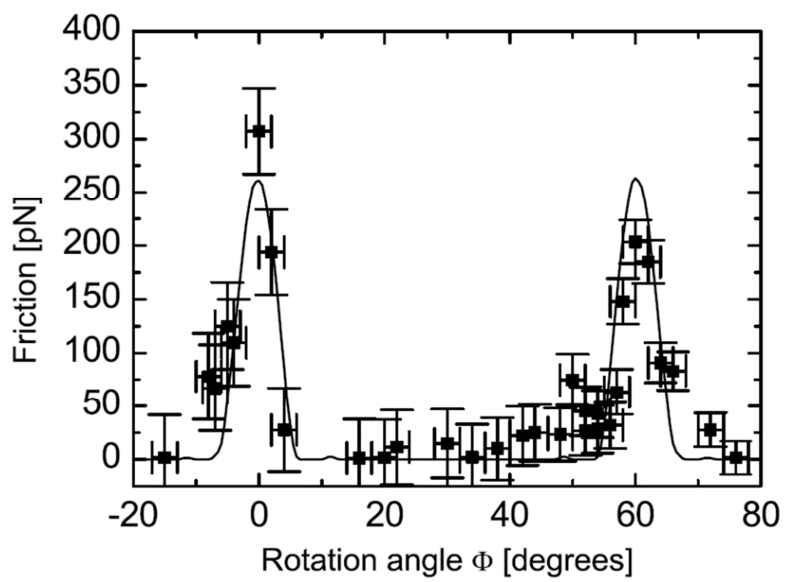

FIGURE 2. Dependency of frictional forces between graphite partners depending on the relative angle of rotation. In directions where the lattices are commensurate, there is large friction. In the uncommensurate orientations, the friction practically does vanish. Source: [9]. 


\section{ADHESION CONTRIBUTION TO FRICTION FOR HARD, AMORPHOUS, ELASTIC SMOOTH SURFACES}

The problem is that hard, amorphous, elastic smooth surfaces are elastic, so they show no plasticity and no viscosity, they are amorphous and thus intrinsically incommensurate to each other and they are smooth which can be shown is equivalent to high enough later stiffness of individual asperities to prevent elastic instabilities. Thus, there is no energy dissipation due to plasticity, no due to viscosity and no due to elastic instabilities. But how then can appear final friction which we do observe?

Let us again state it clearly: If a system is conservative and can be transferred from one point to the other quasistatically, slow, without sudden jumps, than it is necessarily friction free. Discontinuities, jumps on some scale are the key for understanding friction. This is most important fundamental idea in understanding friction. Hard, elastic, smooth, amorphous, surfaces show no instabilities - with one exception - if they attract each other with adhesion forces. And we know that all neutral bodies do attract each other with Wan der Waals forces. We thus discuss in the following adhesive forces and adhesive contribution to friction.

In some sense, adhesive contribution to friction is very generic one. To move the body along each other, one has to destroy interatomic bonds, which needs energy. In this sense, any frictional force is finally due to adhesion as suggested very early by Tomlinson [10]. But where the dissipation comes from in an adhesive contact?

Let us look at the whole process of indenting and destroying an adhesive contact. First, the bodies do not touch each other and the force is zero. At the very first moment of touch, the configuration is changed abruptly and an adhesive neck appears (Fig. 2). Then we have the process of continuous indentation, which is completely reversible. Under reversing the direction of movement, the system moves along exactly the same line until it comes to the point of instability where the contact is lost at once. Only due to sharp jumps is the energy lost irreversible $($ see $[11,12])$.

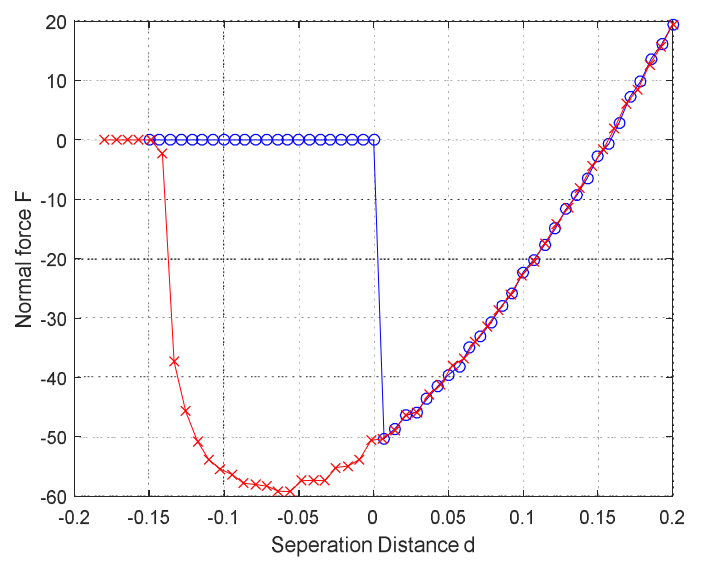

FIGURE 3. Force-distance diagram for an adhesive contact between two parabolic shaped bodies [11,1212]. Blue line: approach, red line: detachment.

Very important: It does not matter whether the contact is formed and destroyed during a normal movement or during a tangential movement (Fig. 4) Due to the adhesive hysteresis, the whole contact history becomes asymmetric with respect to the direction of motion. This leads to the appearance of final macroscopic friction force: Adhesion leads to "adhesion instabilities" [13].

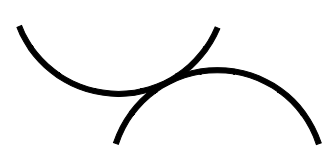

a

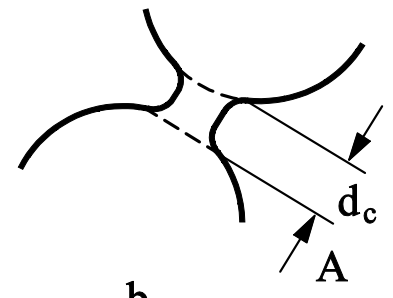

b

FIGURE 4. Creation and destruction of an adhesive neck in tangential movement of asperities [13] 
Let us now look at contacts with a more complicated shape than circular ones. If the shape is smooth, than instability occurs only once in the last contact configuration before complete detachment. But if the form is complicated, this can occur in many steps [14]. If we have two rough surfaces, then there is a plenty of such instabilities. The whole detachment process consist of pinning and jumps of the boundary of the contact, which can be seen in simulation using Boundary Element Method for adhesive contacts as described in [14,15] (Fig. 5).
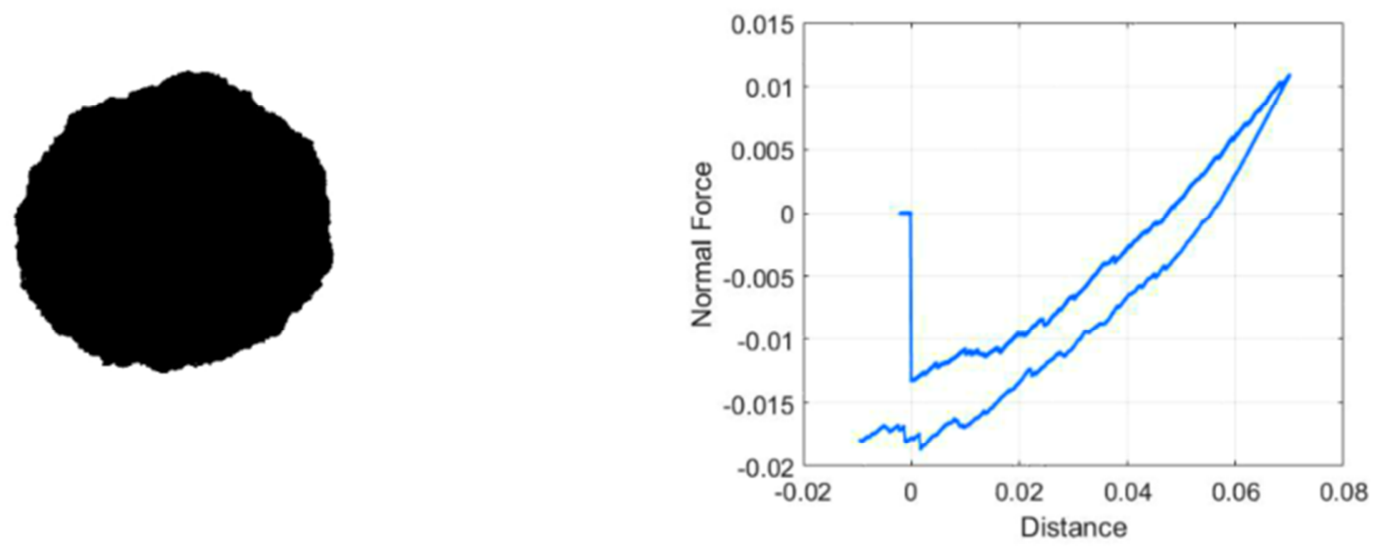

FIGURE 5. On the left: A snapshot of a contact showing a "rough boundary"; on the right: the force-displacement raleation. Due to multiple "micro instabuilities" there is no reversable part of the dependency.

Let us look the same process in experiment (Fig. 6). If contacting surfaces are rough, then the contact area is non-circular. And it does not develop continuously but shows multiple small instabilities, jumps. Each jump is irreversible and during each jump, energy is lost. The energy losses lead to the effect that the indentation and detachment curves do not coincide even in the stage of formed adhesive contact: theoretical results are shown in Fig.5 and experimental in Fig. 6. According to the JKR theory, the system must jump into contact and then move forth and back on the same curve. But in reality the indentation differs from the detachment curve at all distances. This is due to the multiple instabilities (which also can be interpreted as friction in the boundary line.)

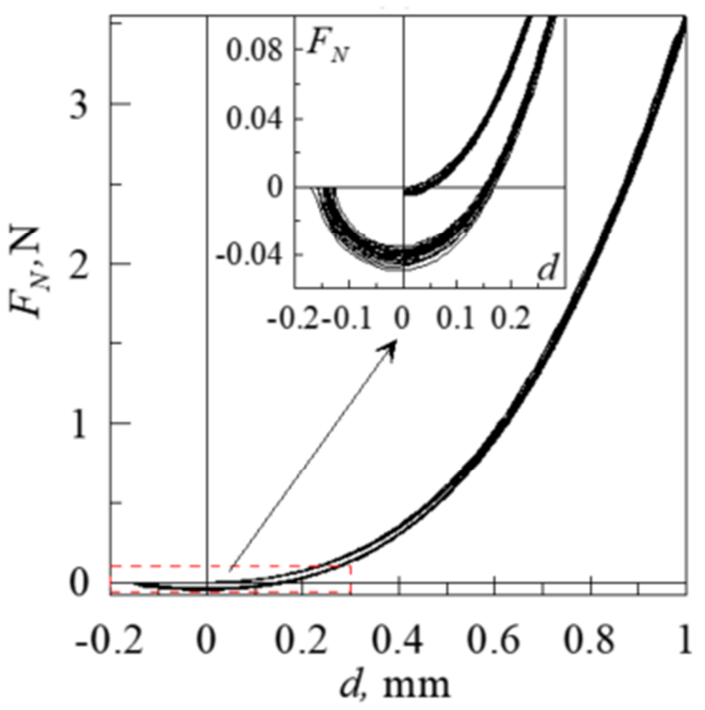

FIGURE 6. Force-displacement relation measured by quasistatic approach and detachment. The upper courve - approach, the lower curve - detachment. Experimental results provided by Ya. Lyashenko, Technische Universität Berlin. 
Absolutely the same effect must appear if we move the contact in tangential direction. And this is seen both in numerical simulations and experiment. Indeed, tangential movement is in some since a superposition of approach and detachment at the same time. Thus, the whole system dissipates energy and friction emerges.

The last question which we would like to address is: what are the governing parameters determining friction? We have just seen that the processes leading to friction in contacts with adhesion are the same. So let us first look at the pure adhesion of rough surfaces. Let us consider the simplest art of roughness - a regular waviness. For macroscopically flat, wavy surfaces, the answer was given by Kenneth Johnson in 1995 [16]. He has shown that if the surfaces have too large roughness, then they are in contact only on the tops of asperities. But if roughness is smaller than some critical value, then the spontaneously jump into complete contact. The critical condition is defined by the Johnson parameter, $\alpha$, which contains the wavelength of waviness, $\lambda$, the specific work of adhesion, $\gamma$, the amplitude of the waviness, $h$, and the effective elastic modulus, $E^{*}$ :

$$
\alpha=\left(\frac{2 \lambda \gamma}{\pi^{2} h^{2} E^{*}}\right)^{1 / 2}
$$

Numerical simulation of adhesive contact of rough surfaces show that the force-displacement relation and in particular, the force of adhesion do depend on roughness. Additional statistical analysis shows that the only parameter, which the force of adhesion does depend on, is the Johnson parameter (Fig. 7) [17].

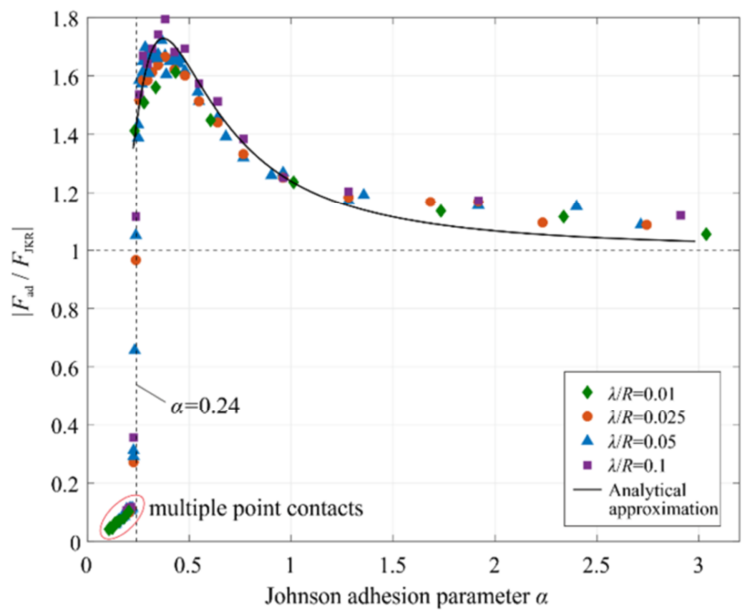

FIGURE 7. Dependency of the normalized force of adhesion on the Johnson parameter obtained in [17] using Boundary Element Method as described in $[14,15]$.

Analysis of simplified asperity models confirms that it is basically the Johnson parameters which completely characterizes adhesion of rough surfaces [13]. We thus hypothesize that it is the Johnson parameter, which determines the frictional force, too. However, for determining of the corresponding "mastrer curve" additional numerical studies are necessary.

\section{CONCLUSION}

In this paper, we argued that adhesive contbution to friction is a very fundamental one and does exist even in very hard, smooth, amorphus contacts where all other mechanicsms of energy disspation are suppressed. This mechanicsm does already exist in normal contact: due to the roughness of surfaces, the contact area is changing not continuously but by jumps, while each jump is related to some energy dissipation. The same effect is seen in tangential movement: both front side and back side of the contact boundry are moving in jumps causing energy dissipation. We argue that the main governing parameter determining the force of friction is in this case the "Johnson parameter" introduced by K.H. Johnson in 1995 [16]. 


\section{ACKNOWLEDGMENTS}

The author acknowledges inspiring discussions with Yakov Lyashenko, and Qiang Li. This work was supported by Deutsche Forschungsgemeinschaft (DFG) and by the Tomsk State University Academic D.I. Mendeleev Fund Program.

\section{REFERENCES}

1. J. Krim, Front. Mech. Eng. 5, 22 (2019).

2. F. P. Bowden and D. Tabor, The Friction and Lubrication of Solids (Clarendon Press, 2001).

3. L. Prandtl, ZAMM 8, 85-106 (1928).

4. V. L. Popov and J.A.T. Gray, ZAMM 92, 683-708 (2012).

5. V. L. Popov and J. A. T. Gray, Prandtl-Tomlinson Model: A Simple Model Which Made History," in The History of Theoretical, Material and Computational Mechanics - Mathematics Meets Mechanics and Engineering, edited by Stein E. (Springer, Berlin, Heidelberg, 2014), pp. 153-168.

6. E. Meyer, R. M. Overney, K. Dransfeld, and T. Gyalog, Nanoscience: Friction and Rheology on the Nanometer Scale (World Scientific, Singapore, 1998)

7. V. L. Popov, Contact mechanics and fricion: physical principles and applications, 2nd ed. (Springer, 2017)

8. A. Socoliuc, R. Bennewitz, E. Gnecco, and E. Meyer, Phys. Rev. Lett. 92, 134301 (2004).

9. M. Dienwiebel et al., Phys. Rev. Lett. 92, 126101 (2004).

10. G. A. Tomlinson, The London, Edinburgh, and Dublin philosophical magazine and journal of science 7, 905 (1929).

11. K. L. Johnson, K. Kendall, and A. D. Roberts, Proc. of the Royal Soc. of London, Series A, 324, 301-313 (1971).

12. V. L. Popov, M. Heß, and E. Willert, Handbook of contact mechanics (Springer, 2019).

13. R. Heise and V. L. Popov, Trib. Lett. 39, 247-250 (2010).

14. V. L. Popov, R. Pohrt, and Q. Li, Friction 5, 308-325 (2017).

15. R. Pohrt and V. L. Popov, Facta Universitatis, Series: Mech. Eng. 13, 3-10 (2015).

16. K. L. Johnson, Int. J. Solids Struct. 32, 423-430 (1995).

17. Q. Li, R. Pohrt, and V. L Popov, Front. Mech. Eng. 5, 7 (2019). 